\title{
Delay Systems Synthesis using Multi-Layer Perceptron Network
}

\author{
D. Plonis*, A. Katkevičius, V. Urbanavičius, D. Miniotas, A. Serackis \\ AND A. Gurskas \\ Vilnius Gediminas Technical University, Department of Electronic Systems, \\ Naugarduko Str. 41-413, LT-03227, Vilnius, Lithuania \\ (Received September 25, 2017; in final form January 2, 2018)
}

\begin{abstract}
The aim of this paper is to accelerate development and investigation of the delay systems. The computational time for investigation of particular design of delay system may take from several minutes up to several days. To achieve the required constructional parameters of the system, the iterative calculations usually should be repeated many times. In this paper, an artificial neural network is proposed to be used as the universal approximator for solving mathematical problems of delay system investigation instead of usual analytical and numerical techniques. The application of a multi-layer perceptron is proposed for approximation of solution space with discrete estimates, which were initially received by application of numerical techniques. Different structures of the multi-layer perceptron were tested for approximation. The difference between delay systems synthesis, which was estimated using numerical techniques and trained multi-layer perceptron did not exceed $5 \%$ for any of the six design parameter values. The execution time for estimating single delay system was reduced from $240 \mathrm{~s}$ to $20 \mathrm{~ms}$. Such fast estimation of design parameters enables performing delay system analysis and design in real time, preserving time for structure visualization in $3 \mathrm{D}$ or virtual reality environment.
\end{abstract}

DOI: 10.12693/APhysPolA.133.1281

PACS/topics: neural networks, delay systems, time consumption, iterative calculations

\section{Introduction}

At present, the meander delay systems (MDSs) are widely used in electronic devices and equipment for signal synchronization [1,2] and filtering [3]. Moreover, meander delay systems are frequently applied as specific meander structures for small-sized antennas $[4,5]$, resonators [6], and other devices [7].

The methods used for analysis of MDSs can be conditionally united in two groups: analytical [8] and numerical [9]. Analytical methods require the compilation of complicated mathematical models. Meanwhile, the numerical methods are used in commercial software packages. For example, an improved u-shaped microstrip meander-line slow wave structure for high efficiency Gband travelling wave tubes was designed using CST Microwave Studio ${ }^{\circledR}$, which is based on the method of finite differences in time domain [10]. Ultra-wide stopband in a compact low pass filter using stepped impedance resonators is designed using Sonnet ${ }^{\circledR}$, which is based on the method of moments [11]. However, the duration of calculations can reach dozens of hours even if modern workstations are used [12]. In this way, the entire design cycle of the delay system could be even longer, as in this first stage of investigation it is necessary to find the optimal design parameters of the MDS through multiple repetitions. Long computation time then becomes a problem. In addition, another problem can arise when

*corresponding author; e-mail: darius.plonis@vgtu.lt the dispersion equation, which in general binds design parameters of the MDS and frequency of analysis, has either no solutions, or several solutions requiring selection of the correct one by using iterative calculations.

Artificial neural networks can be used as the universal approximators [13] for solving various mathematical problems as numerical analysis tool. For example, a modified shaped patch antenna is analyzed using a feed forward back propagation artificial neural network [14]. Power amplifiers were analyzed using a combination of several neural networks [15], each network was used for prediction of certain parameter of an amplifier. Radial basis function network was used for modelling an RF MEMS phase shifter [16]. A multi-layer perceptron (MLP) network was used to optimize parameters of a microstrip patch antenna [17]. Artificial neural networks are also widely used in filter design [18]. It is also possible to use artificial neural networks in different signal processing tasks: investigation of electron optical parameters [19], analysis of radial dependence of the localized magnetic field [20], classification of electron gun operation modes [21]. Artificial neural networks can be also used in combination with other techniques. For example, the inversion of remote sensing data is made using multiple ratios of spectral radiation intensities and artificial neural networks [22]. As one of the main advantages, some researchers [23] suggest abandoning iterative numerical calculations altogether if a correctly trained neural network is available.

In this paper, we propose an algorithm to allow transition from the usual iterative numerical calculations to a solution based on neural networks in the field of investi- 
gation of MDSs. Neural networks should allow avoiding problems that arise by using the usual methods.

The application of the MLP gives a possibility for researchers to view in real time the structure and the dimensions of the designed delay system (DS) as well as link the input parameters of the MLP (characteristic impedance $Z_{0}$, relative permittivity $\varepsilon_{r}$ of the dielectric substrate, and delay time $t_{d}$ ) to the design parameters of the delay system. In addition, sufficiently fast estimation of the design parameters for the DS enables performing a real-time $3 \mathrm{D}$ visualization of the design, suitable for viewing on modern devices, such as virtual reality (VR) headsets.

\section{Materials and method}

The delay system model was used in the investigation. The general model of this system is shown in Fig. 1, and its detailed description can be found in [24]. It was shown in $[25,26]$ that duration of calculations during analysis of the MDSs increases considerably when it is necessary to solve complex dispersion equation. Number of dispersion equations depends on the model of the meander system. This number increases when the meander structure becomes more complex with more internal and external dielectric layers. In this particular example, we have the matrix which consists of ten rows and ten columns. The dispersion equation of the DS always should be a square matrix. The size of the square matrix can vary $m \times n$, where $m$ is equal to $n$ ( $m$ is the number of elements in the row of the matrix, $n$ is the number of elements in the column of the matrix). The size of the square matrix (dispersion equation or determinant) depends on the task and on the difficulty of the delay system.

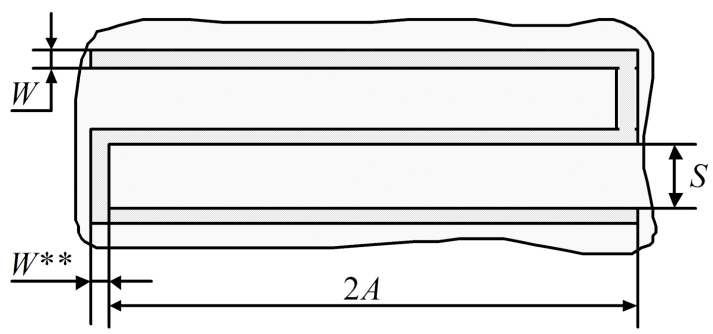

(a)

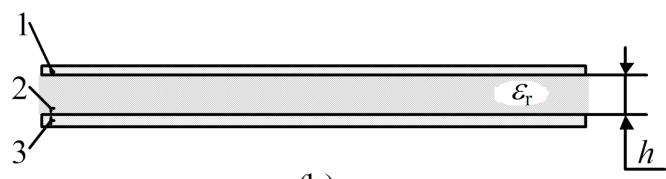

(b)

Fig. 1. The general model of the meander delay system: (a) the top view of meander delay system, and (b) cross-section of the system, where 1 is the dielectric substrate, 2 is the meander shape conductor, 3 is the grounded external shield, $h$ is the thickness of dielectric substrate, $W$ is the width of the meander conductor, $W^{* *}$ is the microstrip connecting adjacent meander stripes, $2 A$ is the length of the conductor, $S$ is the gap between neighbour conductors.
However, the time needed to solve the dispersion equations and to analyse the results may be greatly reduced by using MLP network.

The general algorithm of migration from numerical methods to MLP-based solution and synthesis of delay systems is presented below (Fig. 2). All the procedure can be divided into four stages: collection of delay system samples (1-4 blocks); training of the MLP (5-6); verification of the trained network (7-8); prediction of the DSs design parameters using the trained and verified MLP.

Samples of the DS were collected using traditional iterative numerical synthesis methods in the first stage of the algorithm. At this stage the structure of the DS was selected (1st step). The design parameters $\left(h, W, W^{* *}\right.$, $S, 2 A, n)$ were estimated for the known structure of the DS (2nd step). Here $h$ denotes the thickness of dielectric substrate and upper dielectric, $W$ is the width of the meander conductor, $2 A$ is the length of the conductor, $S$ is the gap between neighbour conductors, $n$ is the number of strips in the meander.

Electrical parameters (characteristic impedance $Z_{0}$, and the delay time $t_{d}$ ) were calculated according to the given design parameters (3rd step). Results were saved to the separated groups for network training and verification (4th step). At the 5th step of the algorithm the structure and initial parameters MLP were set. Selected MLP network was trained using training samples of DS (6th step). Verification procedure was performed using the verification data samples of the DS (7th step). The condition was checked whether the verification has succeeded (8th step). It was necessary to jump to the 2 nd or 5th step (to collect some more data for training or to repeat the training procedure of MLP network), if condition was not satisfied. It is time to use the MLP network for the prediction of design parameters of DS if the condition was satisfied. The prediction of design parameters of DS is called the synthesis of DS.

The structure of MLP network was selected depending on the training data samples of the delay system (5th step of the algorithm). The investigated MLP network had three inputs and six outputs with a single hidden layer. In order to receive optimal approximation results, the structure of the MLP can be adapted by changing the number of neurons in the hidden layer (Fig. 3). During the experimental investigation of the MDS, the size of the hidden layer consists of eight neurons. The activation function of neuron in the hidden layer was sigmoidal. As it is seen in Fig. 3, the type of activation function is presented above each layer of the MLP. A linear activation function was used in the output layer. Therefore, the outputs of MLP were estimated as the weighted sum of hidden layer outputs (Fig. 3).

Together with the desired delay time $t_{\mathrm{d}}$, two additional parameters were used as MLP inputs: the characteristic impedance $Z_{0}$ and the relative permittivity $\varepsilon_{r}$. The desired design parameters of the DS were expected as the outputs of the network. 


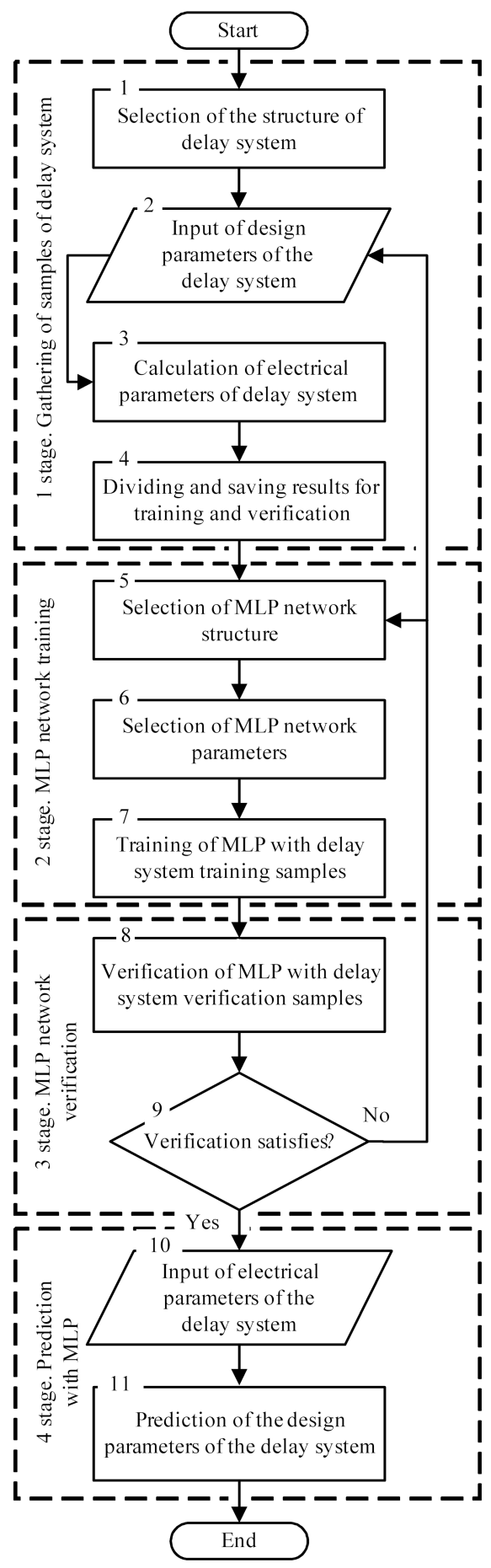

Fig. 2. Algorithm of migration from traditional numerical methods of synthesis of the delay system to neural network methods.

15 meander delay systems samples were collected for the training dataset (see Table I). Every sample consisted of $3 \times 1$ size input and $6 \times 1$ size target vectors. The overall size of the training dataset was $15 \times 9$. The overall size of validation dataset was $4 \times 9$. MLP training was repeated with every sample vector not less than ten times. Best

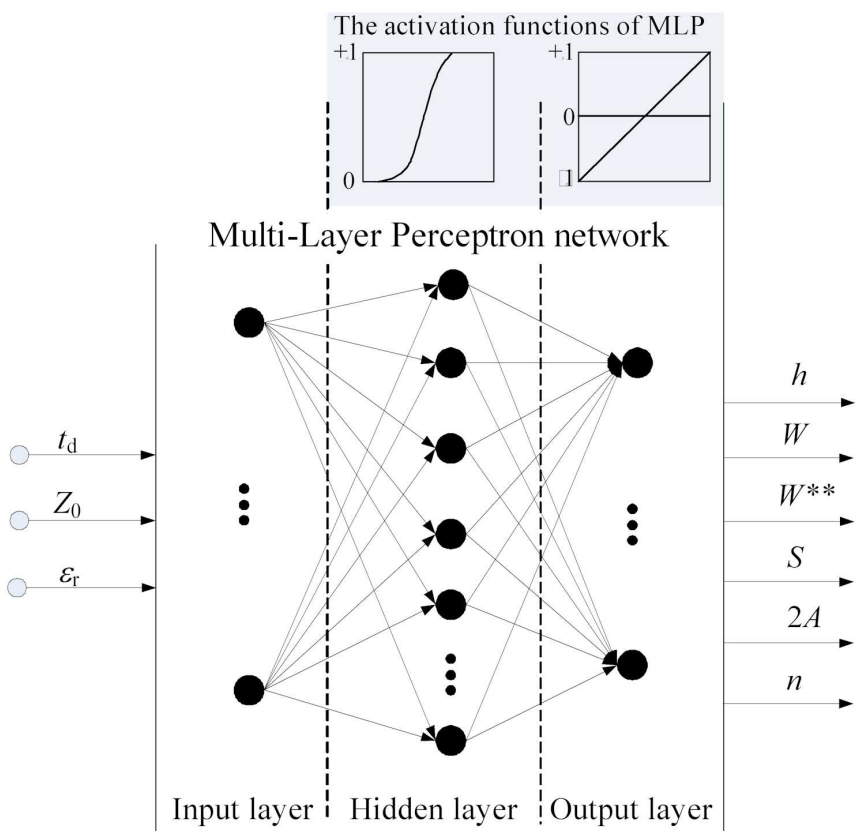

Fig. 3. The structure of multi-layer perceptron network.

matching results comparing with results, obtained using numerical methods, were selected for further processing. A computer experiment performed on the created algorithm (Fig. 2) showed that the proposed algorithm allows quick prediction of design parameters of the delay system with acceptable deviation of the parameter values.

The prediction of design parameters of delay system can be performed, when the vector of delay time $\boldsymbol{t}_{\mathrm{d}}$ consists of $\boldsymbol{t}_{\mathrm{d}}=\left\{t_{\mathrm{d} \min } ; \Delta t_{\mathrm{d}} ; t_{\mathrm{d} \max }\right\}$, where $t_{\mathrm{d} \text { min }}$ and $t_{\mathrm{d} \max }$ are the initial boundaries of the vector of delay time $\boldsymbol{t}_{\boldsymbol{d}}$. The training step $\Delta t_{\mathrm{d}}$ of delay time is also an important parameter. The next important electrical parameter of delay systems is the vector of characteristic impedance $Z_{0}$, which consists of $Z_{0}=\left\{Z_{0 \min } ; \Delta Z ; Z_{0} \max \right\}$, where $Z_{0}$ min and $Z_{0 \text { max }}$ are the initial boundaries of the vector. $\Delta Z_{0}$ is the training step of characteristic impedance. The relative permittivity $\varepsilon_{r}$ of dielectric substrate is constant and equal to 7.3 .

To increase the number of examples for training process of MLP, a radial basis function network (RBF) was used for interpolation of the training values. RBF was used to interpolate values of delay time $\boldsymbol{t}_{\boldsymbol{d}}$ and characteristic impedance $\boldsymbol{Z}_{\mathbf{0}}$. The training process of RBF networks was explained in a previous work by the authors [27]. The training process of multi-layer perceptron network was performed, when initial boundaries of the vector of delay time $\boldsymbol{t}_{\boldsymbol{d}}$ were equal to $t_{\mathrm{d} \min }=5.92 \mathrm{~ns}$; $t_{\mathrm{d} \max }=20.85 \mathrm{~ns}$. The training step $\Delta t_{\mathrm{d}}$ was equal to $\Delta t_{\mathrm{d}}=0.01 \mathrm{~ns}$ and the boundaries of the characteristic impedance vector $Z_{0}$ were $Z_{0} \min =45.60 \Omega$; $Z_{0 \max }=52.90 \Omega$. The training step of the characteristic impedance vector was $\Delta Z_{0}=0.01 \Omega$. 
Results of synthesis of the meander delay system using numerical iterative methods

\begin{tabular}{c|c|c|c|c|c|c|c|c|c|c|c|c|c|c|c}
\hline \hline $\begin{array}{c}\text { Delay } \\
\text { systems } \\
\text { number }\end{array}$ & 1 & 2 & 3 & 4 & 5 & 6 & 7 & 8 & 9 & 10 & 11 & 12 & 13 & 14 & 15 \\
\hline$t_{\mathrm{d}}[\mathrm{ns}]$ & 5.92 & 5.94 & 6.60 & 5.94 & 6.00 & 5.70 & 6.38 & 20.50 & 20.62 & 20.65 & 20.66 & 20.85 & 20.68 & 20.68 & 20.66 \\
$Z_{0}[\Omega]$ & 45.60 & 45.60 & 52.89 & 51.92 & 51.92 & 53.10 & 48.70 & 52.20 & 51.40 & 52.10 & 52.00 & 52.80 & 51.80 & 52.80 & 52.90 \\
$\varepsilon_{r}$ & 7.30 & 7.30 & 7.30 & 7.30 & 7.30 & 7.30 & 7.30 & 7.30 & 7.30 & 7.30 & 7.30 & 7.30 & 7.30 & 7.30 & 7.30 \\
$h[\mathrm{~mm}]$ & 0.478 & 0.478 & 0.468 & 0.452 & 0.452 & 0.472 & 0.441 & 0.518 & 0.511 & 0.516 & 0.518 & 0.511 & 0.511 & 0.521 & 0.524 \\
$W[\mathrm{~mm}]$ & 0.620 & 0.614 & 0.463 & 0.465 & 0.452 & 0.455 & 0.457 & 0.393 & 0.393 & 0.392 & 0.395 & 0.402 & 0.395 & 0.390 & 0.393 \\
$W^{* *}[\mathrm{~mm}]$ & 0.618 & 0.613 & 0.462 & 0.466 & 0.453 & 0.458 & 0.459 & 1.969 & 1.968 & 1.970 & 1.976 & 1.978 & 1.969 & 1.968 & 1.972 \\
$S[\mathrm{~mm}]$ & 0.368 & 0.371 & 0.359 & 0.359 & 0.368 & 0.366 & 0.362 & 0.406 & 0.408 & 0.408 & 0.405 & 0.341 & 0.354 & 0.360 & 0.357 \\
$2 A[\mathrm{~mm}]$ & 18.365 & 18.367 & 18.52 & 15.434 & 15.448 & 15.441 & 15.136 & 16.26 & 16.259 & 16.263 & 16.26 & 16.958 & 16.97 & 16.971 & 16.966 \\
$n$ & 55 & 55 & 67 & 67 & 67 & 32 & 67 & 198 & 198 & 198 & 198 & 200 & 200 & 200 & 200
\end{tabular}

\section{Results and discussion}

In order to compare the prediction results with those of the iterative methods, we have applied the proposed algorithm to the models of the delay system investigated previously by using iterative methods of the commercial software tools. The prediction results were also compared with the results of experimental measurement investigation.

\subsection{Results of prediction of delay systems}

The results obtained using traditional iterative calculations will be compared with the experimental measure- ment results and with those predicted by the MLP network. Prediction of design parameters of delay system is conducted in four different cases:
1. $t_{\mathrm{d}}=6.9 \mathrm{~ns} ; Z_{0}=52.89 \Omega(\mathrm{DS} 1)$.
2. $t_{\mathrm{d}}=6.45 \mathrm{~ns} ; Z_{0}=48.7 \Omega(\mathrm{DS} 2)$.
3. $t_{\mathrm{d}}=20.52 \mathrm{~ns} ; Z_{0}=52.0 \Omega(\mathrm{DS} 3)$.
4. $t_{\mathrm{d}}=20.68 \mathrm{~ns} ; Z_{0}=51.8 \Omega(\mathrm{DS} 4)$.

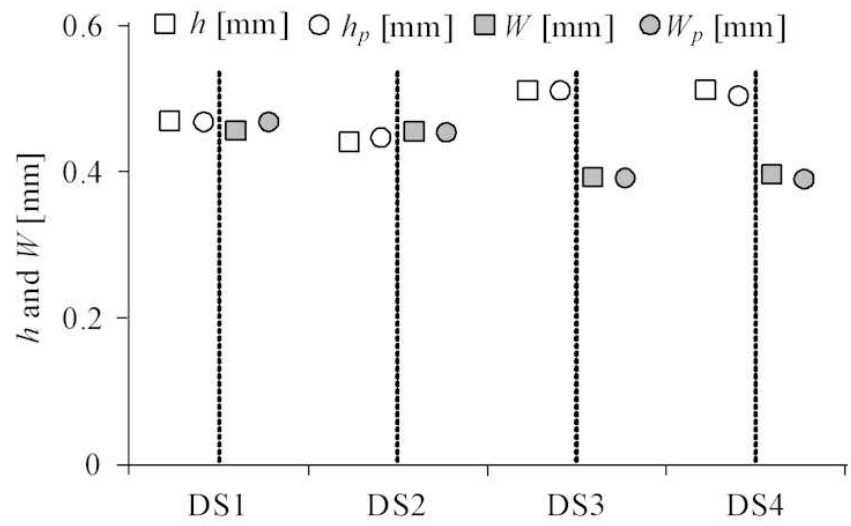

(a)

Investigation case

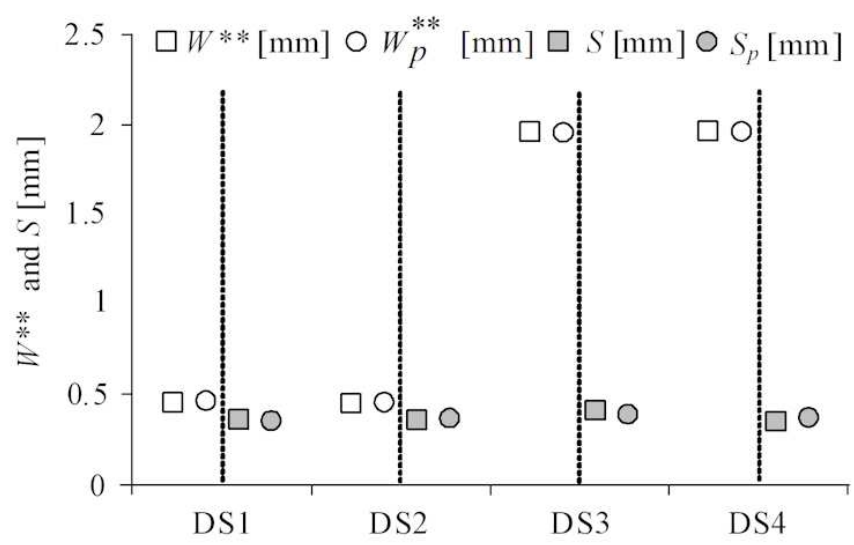

(b)

Investigation case

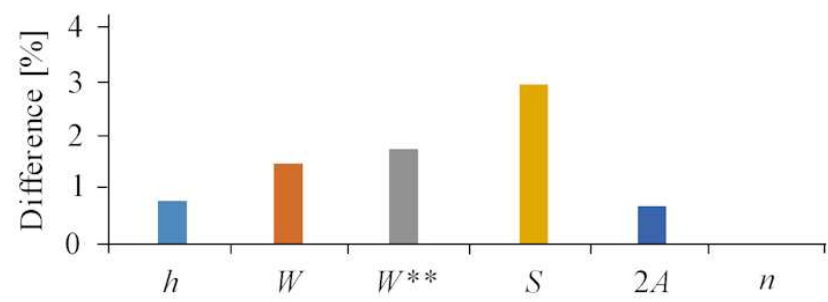

(d)

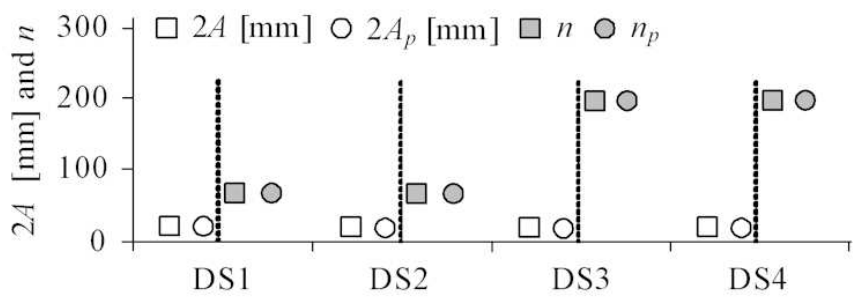

(c)

Investigation case
Parameters

Fig. 4. The comparison of the results predicted and calculated by using iterative methods: (a) comparison of design parameters $h$ and $W,(\mathrm{~b})$ comparison of design parameters $W^{* *}$ and $S$, (c) comparison of design parameters $2 A$ and $n$, (d) difference of all parameters, where lower index $p$ means results of prediction. 
Results of prediction of design parameters of delay system are presented in Fig. 4. The following can be deduced on the basis of the obtained results. The highest difference between the calculated and predicted $S$ and $2 A$ design parameters were received for the high values of $t_{d}$. The difference of calculated and predicted gap between neighbour conductors $S$ was $5 \%$. However, the difference between calculated and predicted width $W$ was $1.3 \%$ for high value of delay time and $3.2 \%$ for low value of delay time $t_{\mathrm{d}}$. It shows an inverse relation between $W$ and $S$ design parameters in delay system. The influence of this difference to the performance of the delay system is small. However, calculations were performed 12,000 times faster.

Calculations using numerical methods and predictions using neural networks of parameters of the DSs were performed on a regular desktop computer with the following parameters: Intel ${ }^{\circledR}$ Core $^{\mathrm{TM}} \mathrm{i} 5-2410 \mathrm{M}$ CPU @ $2.30 \mathrm{GHz}$ processor; 4 GB of RAM; NVIDIA ${ }^{\circledR}$ GeForce ${ }^{\circledR}$ GT $520 \mathrm{M}$ 1 GB RAM video card.

The calculation time was equal to $240 \mathrm{~s}$ using iterative numerical methods. The prediction time was no longer than $0.02 \mathrm{~s}$ using multi-layer perceptron networks. It is 12,000 times faster compared to those obtained using iterative numerical methods. Training of the multi-layer perceptron network took no longer than $3 \mathrm{~s}$. Taking into account that for the selected range of input parameters, training of the MLP should be performed once, the delay system design using design parameters prediction can be performed in real time.

\subsection{Results of experimental investigation}

The setup of experimental investigation of the delay system (Fig. 5) consisted of control computer, sampling oscilloscope, test pulse oscillator, and delay system under test. Measurements were performed with a sampling oscilloscope PicoScope 9300, it was managed by control computer and the experimental investigation results were visualized using the control computer, too.

The real structure of meander delay system (Fig. 6) was manufactured according to the prediction results of design parameters of delay system using multi-layer perceptron network. The meander delay system was predicted when delay time $t_{\mathrm{d}}=20.80 \mathrm{~ns}$, and the characteristic impedance $Z_{0}=50 \Omega$, and the relative permittivity $\varepsilon_{r}$ of dielectric substrate is 7.3. The results of prediction: $h=0.509 \mathrm{~mm} ; W=0.403 \mathrm{~mm} ; W^{* *}=1.978 \mathrm{~mm}$; $S=0.340 \mathrm{~mm} ; 2 A=16.956 \mathrm{~mm} ; n=204$.

The results of measurement of the delay time are presented in Fig. 7. It can be seen that the measured delay time is $t_{\mathrm{d}}=20.875 \mathrm{~ns}$ and the characteristic impedance $Z_{0}$ was not measured. Also it is seen that the signal in the output of delay system is distorted. It can be explained by the fact that the signal in the output reflects from the mismatched end of the meander delay system (Fig. 6).

If we do not take into account the reflections of signal in the output of delay system and the measurement of

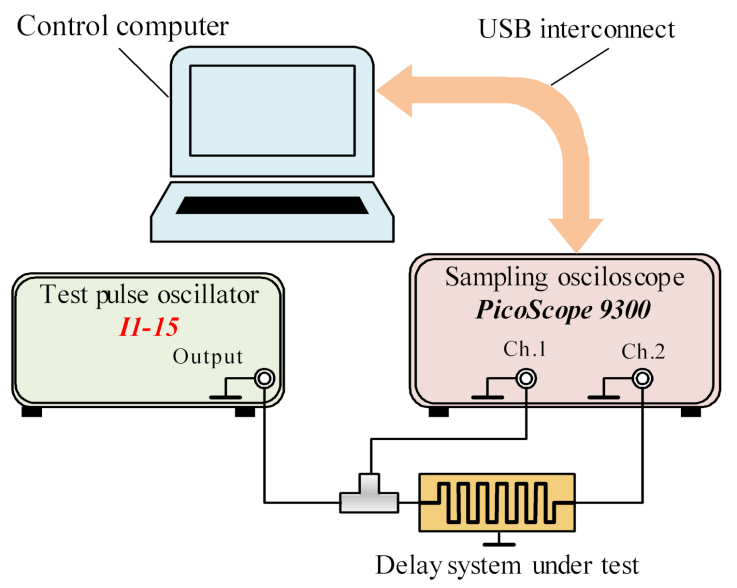

Fig. 5. Setup of experimental investigation of the delay system.

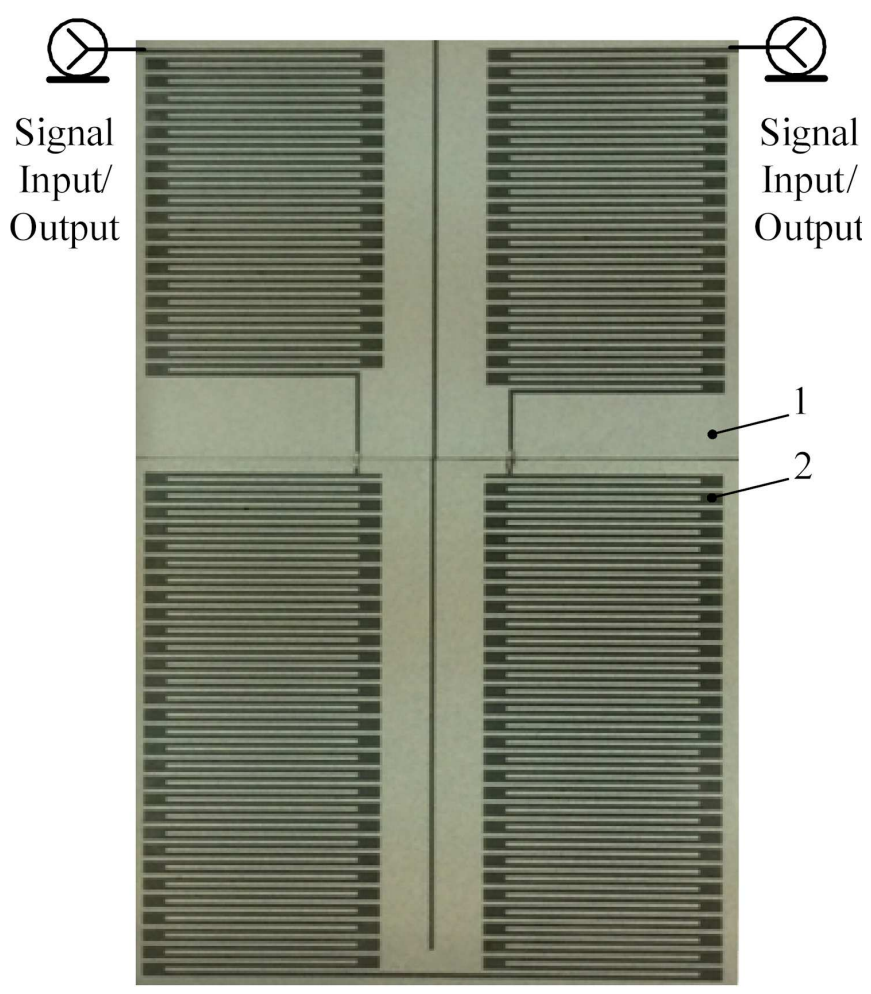

Fig. 6. The structure of the produced meander delay system, where 1 is the dielectric substrate, 2 is the meander shape conductor.

characteristic impedance $Z_{0}$, then the difference between the predicted and measurement results does not exceed 4.83 percent.

\section{Conclusions}

The multi-layer perceptron network can be used for prediction of design parameters of delay systems. MLP network enables considerably a decrease of the time of synthesis of delay systems to the duration acceptable for 


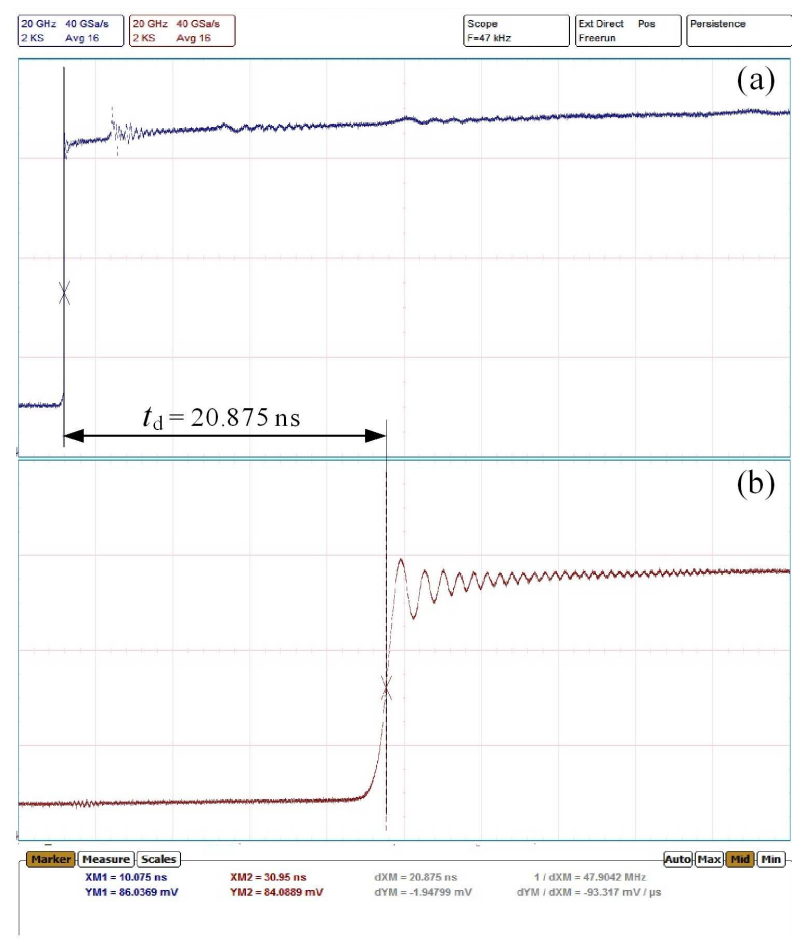

Fig. 7. The measurement results of delay time: (a) input and (b) output signals.

real-time simulations. At the same time, MLP network allows solution of some specific problems arising from the usage of traditional iterative numerical calculations methods.

The proposed synthesis algorithm supports a faster prediction of design parameters of the delay systems by a factor of 12,000 times. The average difference of the predicted and the numerical methods results were not worse than $3.5 \%$ (this maximum difference was received for the gap between neighbour meander conductors). The minimum average difference of the predicted and the numerical methods results were $0 \%$, this difference was received for the number of strips in the meander $-n$. It can be explained by the fact that the number of strips in the meander delay system should have to be rounded to the nearest whole number e.g. $n=204.107$ will be $n=204$.

The difference between the calculated and predicted results varied because of selected learning data sets and the structure of the MLP network. In order to increase the accuracy of prediction it is necessary to select the proper step between data in the learning data sets and also the marginal values of the training data.

The synthesized meander delay system was manufactured and experimentally verified. The average difference of the predicted results, compared to the initial results, obtained using numerical methods, was not more than $5 \%$.

\section{Acknowledgments}

We are very grateful to JSC "Eltesta" for their help in organizing and conducting experimental measurements.
This research was funded by a grant (No. MIP-083/2015) from the Research Council of Lithuania.

\section{References}

[1] M.A. Gubbins, Delay line on a movable substrate accessing data storage media, Seagate Technology Llc., Patent US 12/492,580, US 2013.

[2] T.R. Gazizov, V.K. Salov, S.P. Kuksenko, Wireless Commun. Mobile Comput. 2017, 1965739 (2017).

[3] A. Kumar, D. Kumar Choudhary, R. Kumar Chaudhary, Progr. Electromagn. Res. Lett. 66, 121 (2017).

[4] T. Zhou, Y. Cao, Z. Cheng, M.L. Berre, F. Calmon, Int. J. Anten. Propagat. 2017, 7319275 (2017).

[5] I. Rouissi, J.M. Floch'h, H. Trabelsi, Int. J. Adv. Comput. Sci. Appl. 8, (2017).

[6] M. Renuga Devi, C. Poongodi, D. Deepa, in: Int. Conf. (ICGCCEE), Coimbatore (India), 2014.

[7] S. Mener, R. Gillard, L. Roy, in: 27th Int. Symp. on Antenna Technology and Applied Electromagnetics (ANTEM 2016), Montreal (Canada), 2016.

[8] V. Daškevičius, J. Skudutis, S. Štaras, Elektronika ir elektrotechnika 19, (2013).

[9] A. Gurskas, A. Krukonis, V. Urbanavičius, Elektronika ir elektrotechnika 21, (2015).

[10] E. Tahanian, G.R. Dadashzadeh, Recent Adv. Electric. Electron. Eng. 9, (2016).

[11] L. Murphy, M. Yazdani, E. Arvas, in: IEEE Int. Conf. (ICUWB), Syracuse (USA), 2012.

[12] R. Pomarnacki, A. Krukonis, V. Urbanavicius, Elektronika ir Elektrotechnika 20, (2014).

[13] K. Hornik, Neural Networks 4, (1991).

[14] R. Kaur, A.K. Narula, Int. J. Intellig. Syst. Appl. 9, (2017).

[15] J.J. Michalski, Progr. Electromagn. Res. M 13, (2010).

[16] G.H. Yang, Q. Wu, J.H. Fu, K. Tang, J.X. He, in: IEEE Int. Conf. (INDIN 2008) on Industrial Informatics, 2008.

[17] M. Singhal, G. Saini, Int. J. Comput. Trends Technology 44, (2017).

[18] G. Jain, R.P. Narwaria, Int. J. Eng. Sci. Res. Technol. 6, (2017).

[19] A. Isik, Acta Phys. Pol. A 127, 1317 (2015).

[20] A. Isık, N. Isık, Acta Phys. Pol. A 131, 32 (2017).

[21] N. Isik, A. Isik, Acta Phys. Pol. A 129, 628 (2016).

[22] S. Cięszczyk, Acta Phys. Pol. A 131, 1454 (2017).

[23] A. Serackis, D. Plonis, A. Krukonis, A. Katkevičius, in: Proc. 4th IEEE Workshop, Vilnius (Lithuania), 2016.

[24] V. Urbanavičius, A. Gurskas, R. Martavičius, Elektronika ir elektrotechnika 90, (2009).

[25] A. Katkevičius, S. Štaras, Elektronika ir elektrotechnika 108, (2011).

[26] D. Plonis, A. Katkevičius, V. Mališauskas, A. Serackis, D. Matuzevičius, Acta Phys. Pol. A 129, 414 (2016).

[27] A. Katkevičius, V. Mališauskas, D. Plonis, A. Serackis, Electric. Rev. 88, (2012). 